\title{
COEFICIENTES DE PARTICIÓN DE MERCURIO EN LIXIVIADOS DEL RELLENO SANITARIO LA ESMERALDA
}

Fecha de recepción: 17 de febrero de 2016 • Fecha de Evaluación: 10 de marzo 2016 • Fecha de aceptación: 16 de marzo de 2016

\section{PARTITION COEFFICIENTS OF MERCURY IN SANITARY LANDFILL LEACHATE LA ESMERALDA}

María Fanny Ocampo Barrero, Adela Londoño Carvajal, Gloría Inés Giraldo Gómez*, Nancy Rocío Sanabria González.

\section{RESUMEN}

Se determinó el coeficiente de partición $\left(\mathrm{K}_{\mathrm{d}}\right)$ de mercurio $(\mathrm{Hg})$ en cinco muestras de lixiviado provenientes del relleno sanitario La Esmeralda, de la ciudad de Manizales (Caldas - Colombia). La concentración de mercurio se cuantificó empleando espectroscopia de absorción atómica de vapor frío. Dada la elevada cantidad de materia orgánica presente en los lixiviados y las interferencias por efectos de la matriz, se empleó el método de adición de estándares para validar la metodología usada en la cuantificación de mercurio. El coeficiente de partición de mercurio en los lixiviados entre 222.4 y $3460.2 \mathrm{~L} / \mathrm{kg}$ confirma la composición variable de los mismos, dado el amplio intervalo encontrado en la concentración de Hg total (71.4 - 1336.8 $\mu \mathrm{g} / \mathrm{L}$ ) y sólidos suspendidos (442 - $1310 \mathrm{mg} / \mathrm{L}$ ). Valores de log $\mathrm{K}_{\mathrm{d}}$ entre 2.35 y 2.84 se asocian a muestras de lixiviado en las cuales el $\mathrm{Hg}$ tiende a estar disuelto, en tanto que valores de log $\mathrm{K}_{d}$ entre 3.41 y 3.54 indican mayor afinidad del $\mathrm{Hg}$ a adsorberse sobre los sólidos suspendidos. Cuando el pH del lixiviado disminuye a 6, se modifica la distribución de las especies de mercurio en las dos fases, aumentando la concentración de mercurio disuelto ( $K_{d}$ disminuye). A pH superior a 8, la relación de la concentración de $\mathrm{Hg}$ adsorbido y disuelto permanece constante ( $K_{d}$ no varía).

Palabras clave: Líquido percolado; Agua residual; Movilidad de metales; Adsorción.

Grupo de Trabajo Académico en Ingeniería Hidráulica y Ambiental. Facultad de Ingeniería y Arquitectura, Universidad Nacional de Colombia Sede Manizales, Campus La Nubia.

* Autor Corresponsal. E-mail: gigiraldogo@unal.edu.co 
The partition coefficient $\left(\mathrm{K}_{\mathrm{d}}\right)$ of mercury $(\mathrm{Hg})$ in five samples of leachate from La Esmeralda landfill in the city of Manizales (Caldas - Colombia) was determined. The mercury concentration was measured by using cold vapor atomic absorption spectroscopy. Given the large amount of organic matter found in the leachate as well as interference due to matrix effects, the standard addition method was used to validate the methodology used for quantification of mercury. The partition coefficient of mercury in leachate between 222.4 and $3460.2 \mathrm{~L} / \mathrm{kg}$ confirms its changing composition given the wide range found in the concentration of total $\mathrm{Hg}\left(71.4-1336.8 \mathrm{mg} / \mathrm{L}\right.$ ) and suspended solids (442-1310 mg/L). Log $\mathrm{K}_{\mathrm{d}}$ values between 2.35 and 2.84 are associated with leachate samples in which $\mathrm{Hg}$ tends to be dissolved, while log $\mathrm{K}_{d}$ values between 3.41 and 3.54 indicate a higher affinity of $\mathrm{Hg}$ to get adsorbed on the suspended solids. When the $\mathrm{pH}$ of the leachate decreases to 6 , the distribution of mercury species in the two phases appears changing, increasing the concentration of dissolved mercury ( $K_{d}$ decreases). At a pH above 8 , the concentration ratio of $\mathrm{Hg}$ adsorbed and dissolved remains constant $\left(K_{d}\right.$ unchanged).

Key words: Percolate liquid, Wastewater; Mobility of metals, Adsorption.

\section{INTRODUCCIÓN}

Un relleno sanitario es un lugar técnicamente diseñado para la disposición final de los residuos sólidos urbanos en el suelo. Los criterios de calidad ambiental de un relleno sanitario están determinados por el tratamiento de los lixiviados, el control de olores desagradables, el impacto en la emisión de gases, el manejo de la cobertura de los residuos sólidos allí dispuestos y el riesgo para la salud por la reproducción de vectores (Noguera y Olivero, 2010; Fang et al, 2012). La descomposición de los residuos en rellenos sanitarios mediante procesos fisicoquímicos y biológicos genera como subproductos biogas y un líquido residual denominado lixiviado, el cual contiene agua proveniente de la humedad inicial del residuo, de las reacciones propias del sistema (disolución de compuestos presentes en el residuo y degradación de la materia orgánica) y de la infiltración de la lluvia que cae sobre el área del relleno (Peng, 2013; Raghab et al, 2013).
El flujo y la composición de los lixiviados en los rellenos sanitarios es variable (Calace, 2001; Renoua et al, 2008) y normalmente se caracterizan por tener un $\mathrm{pH}$ ácido o básico dependiendo de la edad del relleno, una elevada carga de materia orgánica, ácidos fúlvicos y húmicos (Fan et al, 2006; Silva et al, 2013), compuestos nitrogenados (Calace, 2001), metales pesados (Hennebert et al, 2013) y sales inorgánicas (Žgajnar Gotvajn et al, 2011). Metales como cadmio, niquel, zinc, cobre, cromo, plomo y mercurio son encontrados en niveles moderados y algunos pueden estar acomplejados con la materia orgánica disuelta (Claret et al, 2011).

El mercurio $(\mathrm{Hg})$ es uno de los elementos más tóxicos que afectan la salud humana y los ecosistemas. El mercurio en el medio ambiente proviene de fuentes naturales o antropogénicas, incluida la producción de: lámparas fluorescentes, pantallas de cristal líquido, baterías, cloro gaseoso e hidróxido 
de sodio mediante electrólisis en celdas de mercurio y en la minería de oro y plata, entre otros (Leopold et al, 2010; Date et al, 2013; SaldarriagaIsaza et al, 2015; da Cunha et al, 2016). Por lo anterior, es común encontrar mercurio en los lixiviados de los rellenos sanitarios.

Existe una amplia gama de especies de mercurio en nuestro entorno y la forma química del mercurio controla su biodisponibilidad, transporte, persistencia e impacto en el cuerpo humano (Leopold et al, 2010). Por consiguiente, algunos compuestos de mercurio tienen la capacidad de adsorberse sobre el material particulado presente en el agua, y otros compuestos pueden estar disueltos en la fase acuosa o ser liberados a la atmosfera. La mayoría de los compuestos inorgánicos de $\mathrm{Hg}(\mathrm{I})$ son poco solubles en agua y por lo tanto se encuentran principalmente en suelos y sedimentos. El mercurio divalente $\mathrm{Hg}$ (II) puede estar acomplejado con compuestos clorados $\left(\mathrm{HgCl}_{2}, \mathrm{HgCl}^{+}, \mathrm{HgCl}_{4}^{2-}, \mathrm{HgOHCl}\right)$ los cuales son muy solubles en agua, o como hidróxidos $\left(\mathrm{Hg}(\mathrm{OH})_{2^{\prime}}\right.$ $\mathrm{HgO}$ ) y sulfuros de mercurio $(\mathrm{HgS})$ que precipitan. El Hg(II) puede sufrir metilación formando el monometilmercurio $\left(\mathrm{CH}_{3} \mathrm{Hg}^{+}\right.$, soluble en agua) y dimetilmercurio $\left(\left(\mathrm{CH}_{3}\right)_{2} \mathrm{Hg}\right.$, insoluble en agua y muy volátil). Las reacciones de metilación son reversibles, lo cual facilita la movilidad de los compuestos de mercurio (Žagar et al, 2007; Leopold et al, 2010).

La concentración de mercurio en fase acuosa y en el material particulado depende de factores como el pH, el contenido de materia orgánica, la concentración de iones como los cloruros, la actividad microbiana y los equilibrios de adsorción y desorción (Poulain et al, 2007; Leopold et al, 2010), entre otros. Por tanto, algunos compuestos de mercurio son retenidos por las partículas y otros permanecen en solución, relación que puede ser expresada mediante el coeficiente de partición $K_{d}$ (Allison y Allison, 2005)

El relleno sanitario La Esmeralda está ubicado en la ciudad de Manizales (Caldas - Colombia) y en él se disponen alrededor de 450 ton/día de residuos sólidos urbanos. Aunque el relleno sanitario cuenta con sistemas de tratamiento para los lixiviados, la concentración de mercurio total en el efluente varía entre 1.090 y $4.340 \mathrm{mg} / \mathrm{L}$, con un promedio de $2.547 \mathrm{mg} / \mathrm{L}$ (Morales, 2007), valor que excede el límite actual de vertimiento de $0.02 \mathrm{mg} / \mathrm{L}$ estipulado en la legislación nacional (Ministerio de Ambiente y Desarrollo Sostenible, 2015).

Dadas las particularidades de los compuestos de mercurio y la gran movilidad en el suelo-agua-aire, el conocimiento del coeficiente de partición es un parámetro fisicoquímico que cuantifica la afinidad del mercurio por el material particulado o su disolución en medio acuoso. Aunque se ha determinado el coeficiente de partición de mercurio en suelo/agua y sedimentos/agua en ríos y lagos, en lixiviados de rellenos sanitarios no se dispone de esta información, la cual es esencial en la predicción del transporte de esta especie. En este trabajo se determinó el coeficiente de partición de mercurio en lixiviados provenientes del relleno sanitario La Esmeralda y su variación con el contenido de sólidos y el $\mathrm{pH}$ de la muestra, dado que estos dos parámetros presentan variabilidad según la edad del relleno y la etapa en la descomposición de los residuos sólidos. Es importante resaltar que el coeficiente de partición es la base para la selección de un adecuado sistema de post-tratamiento del mercurio presente en los lixiviados, el cual permita cumplir con las concentraciones exigidas por la ley y asegure un residuo líquido inofensivo para la salud humana y el medio ambiente.

\section{MATERIALES Y MÉTODOS}

\section{Reactivos}

Todos los reactivos utilizados para cuantificación del mercurio fueron grado analítico: borohidruro de sodio $\left(\mathrm{NaBH}_{4}, 96 \%\right)$, ácido sulfúrico $\left(\mathrm{H}_{2} \mathrm{SO}_{4}, 98 \%\right), \mathrm{HNO}_{3}(65 \%)$, permanganato 
de potasio $\left(\mathrm{KMnO}_{4^{\prime}}\right.$ 99.3\%), persulfato de potasio $\left(\mathrm{K}_{2} \mathrm{~S}_{2} \mathrm{O}_{8^{\prime}}\right.$ 98\%) nitrato de mercurio $\left(\mathrm{Hg}\left(\mathrm{NO}_{3}\right)_{2^{\prime}}>98 \%\right)$, hidróxido de sodio $(\mathrm{NaOH}, 98.4 \%)$, hidroxilamina sulfato $\left(\mathrm{NH}_{2} \mathrm{OH}\right)_{2} \cdot \mathrm{H}_{2} \mathrm{SO}_{4^{\prime}}$,99\%) y cloruro de sodio $(\mathrm{NaCl}$, $>99 \%$ ). El agua utilizada fue destilada y desionizada.

\section{Recolección de los lixiviados y caracterización}

El relleno sanitario La Esmeralda genera un promedio de $2.16 \mathrm{~L} / \mathrm{s}$ de lixiviados en invierno y 0.98 $\mathrm{L} / \mathrm{s}$ en verano, los cuales son tratados en un sistema biológico anaerobio, compuesto por un tanque homogeneizador y dos reactores anaerobios de manto lodo de flujo ascendente conocidos como UASB (upflow anaerobic sludge blanket), donde la carga orgánica se reduce en un 85\% (Londoño-Carvajal et al, 2014). Las muestras para la caracterización y determinación del coeficiente de partición fueron tomadas a la salida del sistema de tratamiento anaerobio UASB teniendo la precaución de no airearlas y conservar las condiciones anaerobias, posteriormente fueron llevadas al laboratorio para el análisis de: $\mathrm{pH}$, color, conductividad, materia orgánica expresada como demanda química de oxígeno (DQO) y demanda biológica de oxígeno $\left(\mathrm{DBO}_{5}\right)$, sólidos suspendidos totales (SST), cloruros ( $\mathrm{Cl}$ ) y mercurio, siguiendo los métodos estándar de análisis (APHA et al, 2005).

\section{Cuantificación de mercurio \\ Validación de la metodología}

La concentración de Hg se determinó con un espectrofotómetro de absorción atómica Perkin Elmer 3110 con accesorio para generación de vapor frío MH 9-10, técnica conocida como CV-AAS (Cold Vapor - Atomic Absorption Spectrometry), con borohidruro de sodio como agente reductor, argón grado analítico 99.999\% de pureza, lámpara de cátodo hueco Perkin Elmer operada a 6 mA y a 253.7 nm.

Debido a la alta carga de materia orgánica que tienen los lixiviados, se realizó una modificación en el proceso de digestión y dilución para garantizar resultados reproducibles. Se tomaron $10 \mathrm{~mL}$ de la muestra del lixiviado y se diluyeron con agua destilada y desionizada a $100 \mathrm{~mL}$, se adicionaron $5.0 \mathrm{~mL}$ de $\mathrm{H}_{2} \mathrm{SO}_{4}$ concentrado y $2.5 \mathrm{~mL}$ de $\mathrm{HNO}_{3}$ concentrado, se mezcló la solución y se adicionaron $15 \mathrm{~mL}$ de $\mathrm{KMnO}_{4}$ al $50 \% \mathrm{w} / \mathrm{v}$, hasta que el color rosado persistió mínimo 15 minutos. Luego se adicionaron $8.0 \mathrm{~mL}$ del persulfato de potasio al $5 \% \mathrm{w} / \mathrm{v}$ y se sometió la solución a digestión en un baño maría por 2 horas a $95^{\circ} \mathrm{C}$. Finalmente, la solución se enfrío y se agregaron $4.0 \mathrm{~mL}$ de hidroxilamina sulfato - cloruro de sodio al 50\% w/v y se aforó a $200 \mathrm{~mL}$. Para la curva de calibración se tomaron alícuotas de $5 \mathrm{~mL}$ y se adicionó la cantidad de mercurio patrón de 100 rg/L necesario para tener concentraciones en la solución final de 0, 2, 4, 6, 8 y $10 \mu \mathrm{g} / \mathrm{L}$, se aforó la solución a $50 \mathrm{~mL}$ y se determinó la concentración de mercurio.

Para validar la metodología se preparó una matriz de agua residual doméstica, la cual contiene una gran cantidad y variedad de sustancias disueltas y suspendidas, además de materia orgánica fácilmente oxidable. El agua residual doméstica utilizada fue previamente caracterizada y se le adicionó una cantidad de solución patrón de mercurio hasta lograr una concentración de $200 \mu \mathrm{mg} / \mathrm{L}$ de $\mathrm{Hg}$. Las muestras se sometieron al mismo procedimiento que las muestras de lixiviado. Con el fin de eliminar posibles interferencias por efectos de matriz, la concentración de mercurio se evaluó mediante el método de adición de estándares (Harris, 2007; Ellison y Thompson, 2008). Con los resultados de 8 réplicas se determinaron los parámetros estadísticos: promedio, rango, desviación estándar, desviación estándar relativa y error relativo promedio.

\section{Determinación del coeficiente de partición de mercurio $K_{d}$}

Para la determinación del coeficiente de partición $\mathrm{K}_{\mathrm{d}^{\prime}}$ se midió la concentración de mercurio por triplicado a cinco muestras de lixiviado sin filtrar 
(Hg total, $\mu \mathrm{g} / \mathrm{L}$ ) y después de filtración ( $\mathrm{Hg}$ disuelto, $\mu \mathrm{g} / \mathrm{L})$. En ambos casos se realizó digestión de la materia orgánica. El coeficiente de partición se calculó mediante la ecuación (1) (Allison y Allison, 2005):

$$
K_{d}\left(\frac{L}{k g}\right)=\frac{\begin{array}{c}
\text { concentración de Hg adsorbida } \\
\text { por los sólidos suspendidos }\left(\frac{\mu g}{\mathrm{~kg}}\right)
\end{array}}{\text { concentración de Hg disuelto }(\mu \mathrm{g} / \mathrm{L}))}
$$

La concentración de Hg adsorbida corresponde a la concentración de Hg retenida por los sólidos suspendidos ( $\mathrm{Hg}$ total - Hg disuelto, $\mu \mathrm{g} / \mathrm{L}$ ) dividida entre la concentración de sólidos suspen$\operatorname{didos}(\mathrm{kg} / \mathrm{L})$.

Como las concentraciones disuelta y adsorbida de Hg representan fracciones fijas de la concentración total, y estas a su vez dependen del coeficiente de partición $K_{d}(L / k g)$ y la concentración de SST $(\mathrm{kg} / \mathrm{L})$, es posible determinar las fracciones disueltas $\left(F_{\text {dis }}\right)$ y adsorbida $\left(F_{\text {ads }}\right)$ en cada uno de los ensayos mediante las ecuaciones (2) y (3) (Thomann y Di Toro, 1983; ANLA, 2013):

$$
\begin{gathered}
F_{d i s}=\frac{1}{1+K_{d} \times S S T} \\
F_{a d s}=\frac{K_{d} \times S S T}{1+K_{d} \times S S T}
\end{gathered}
$$

A una muestra de lixiviado que presentaba una elevada concentración de sólidos suspendidos se le ajustó el pH a 6, 7, 8 y 9, valores que se presentan con frecuencia en los lixiviados de los rellenos sanitarios en sus diferentes etapas. Para ajustar el pH en el lixiviado se emplearon soluciones $2.0 \mathrm{M}$ de ácido sulfúrico e hidróxido de sodio, y posteriormente se les midió la cantidad de mercurio presente en muestra filtrada y sin filtrar y con los resultados se determinó el coeficiente de partición.

\section{RESULTADOS Y DISCUSIÓN}

\section{Caracterización del lixiviado}

Los resultados de la caracterización físico-química de las muestras de lixiviados del relleno sanitario La Esmeralda se muestran en la Tabla 1.

Tabla 1. Características fisicoquímicas de los lixiviados del relleno sanitario La Esmeralda.

\begin{tabular}{cc}
\hline Parámetro & $\begin{array}{c}\text { Rango encontrado } \\
\text { en el lixiviado }\end{array}$ \\
\hline $\mathrm{pH}$ & $8.03-8.50$ \\
\hline Color $(\mathrm{U} . \mathrm{Pt}-\mathrm{Co})$ & $204.9-2141$ \\
\hline Turbiedad $(\mathrm{NTU})$ & $259-347$ \\
\hline Conductividad $(\mu \mathrm{S} / \mathrm{cm})$ & $13650-18560$ \\
\hline $\mathrm{DOO}\left(\mathrm{mg} \mathrm{O}_{2} / \mathrm{L}\right)$ & $4659-10880$ \\
\hline $\mathrm{DBO}(\mathrm{mg} \mathrm{O} / \mathrm{L})$ & $1365-3150$ \\
\hline $\mathrm{SST}(\mathrm{mg} / \mathrm{L})$ & $442-1310$ \\
\hline $\mathrm{Cl}^{-}(\mathrm{mg} / \mathrm{L})$ & $12800-21646$ \\
\hline $\mathrm{Hg}_{\mathrm{total}}(\mu \mathrm{g} / \mathrm{L})$ & $71.40-1336.80$ \\
\hline
\end{tabular}

Los lixiviados presentan un $\mathrm{pH}$ ligeramente alcalino, característico de un relleno en fase metanogénica, donde predomina la degradación anaeróbica (Kjeldsen et al, 2002). Durante el proceso de generación de lixiviados, estos arrastran gran cantidad de sólidos disueltos y en suspensión contenidos en los residuos sólidos, de allí los elevados valores de color (asociado principalmente a sólidos 
suspendidos), turbiedad (asociada con partículas de tamaño entre 0.1 y $1.0 \mu \mathrm{m}$ ) y conductividad debida a iones disueltos como por ejemplo los cloruros (Londoño-Carvajal et al, 2014). La relación $\mathrm{DBO}_{5} / \mathrm{DQO}$ es un parámetro que permite clasificar los cambios en la composición de los lixiviados de acuerdo a la edad del relleno. Para los lixiviados del relleno sanitario La Esmeralda se obtuvo una relación $\mathrm{DBO}_{5} / \mathrm{DQO}$ cercana a 0.29 , la cual se asocia con un lixiviado intermedio $\left(\mathrm{DBO}_{5} / \mathrm{DQO}\right.$ entre $0.1 \mathrm{y}$ 0.5) proveniente de un relleno de entre 5 y 10 años con biodegradabilidad media (Alvarez-Vazquez et al, 2004; Peng, 2013). Aunque el relleno sanitario La Esmeralda tiene más de 20 años de funcionamiento, el sistema de tratamiento de lixiviados sólo se implementó desde el año 2006, con base en resultados de las investigaciones realizadas en la Universidad Nacional de Colombia Sede Manizales. La concentración de metales de interés ambiental reportada durante el año 2007 en el lixiviado del relleno sanitario La Esmeralda fue la siguiente: plo$\mathrm{mo}(0.04-0.150 \mathrm{mg} / \mathrm{L})$, cromo $(0.030-0.250 \mathrm{mg} / \mathrm{L})$, mercurio (1.090 - $4.340 \mathrm{mg} / \mathrm{L})$ y cadmio (0.010 $0.020 \mathrm{mg} / \mathrm{L}$ ). De los metales evaluados, el mercurio es el que presenta mayor toxicidad y riesgo para la salud humana y el medio ambiente, de allí el interés de evaluar la afinidad del mercurio por el material suspendido o su disolución en medio acuoso.

\section{Validación de la metodología de cuantificación de mercurio en lixiviados}

La caracterización del agua residual doméstica utilizada para la validación de la metodología presentó una DQO de $250 \mathrm{mg} / \mathrm{L}$, sólidos suspendidos totales de $430 \mathrm{mg} / \mathrm{L}$ y ausencia de mercurio. A la muestra de agua residual doméstica se le adicionó $\mathrm{Hg}(0,2,4,6,8$ y $10 \mu \mathrm{g} / \mathrm{L})$ y la calibración del método se realizó empleando el método de adición de estándares (Harris, 2007; Ellison y Thompson, 2008). En la Figura 1 se muestra la curva de calibración de

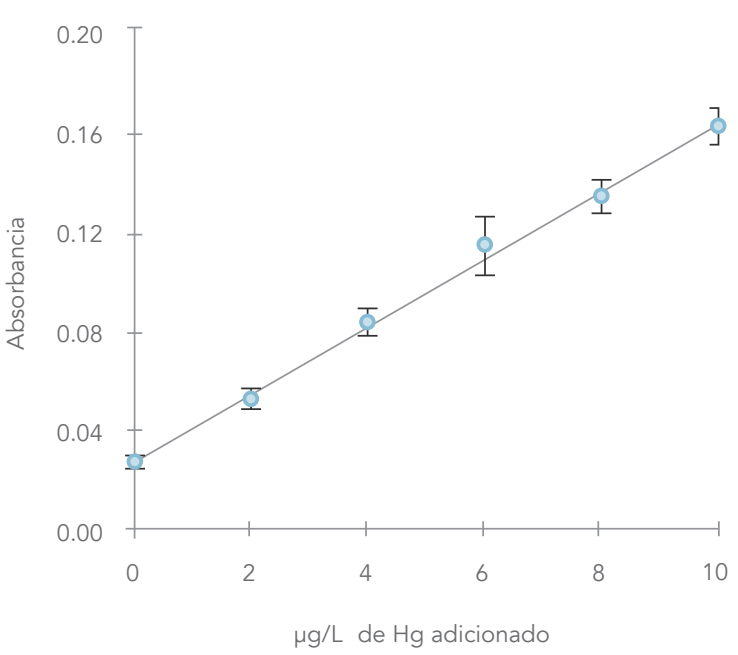

Figura 1. Curva de calibración de mercurio por el método de adición de estándares.

mercurio con el promedio y las desviaciones obtenidas para cada uno de los puntos.

Los parámetros estadísticos para el método modificado en 8 muestras a las que se les adicionó mercurio en una concentración de $200 \mu \mathrm{g} / \mathrm{L}$ fueron: promedio $=201.89 \mu \mathrm{g} / \mathrm{L}$, error relativo promedio $=$ $0.95 \%$, rango $=190.0$ a $214.7 \mu \mathrm{g} / \mathrm{L}$, desviación estándar $=9.06$, desviación estándar relativa $=4.49 \%$ y error relativo promedio $=0.95 \%$. Una vez hecha la validación del método modificado se midió la concentración de mercurio total para una muestra de lixiviado y los resultados del análisis de 4 réplicas obtenidos fueron: media $=791.71 \mu \mathrm{g} / \mathrm{L}$, desviación estándar $=26.10$, desviación estándar relativa $=$ $3.30 \%$. En todos los casos los coeficientes de correlación de las curvas de calibración fueron superiores al $99.5 \%$.

\section{Determinación de coeficiente de partición}

En la Tabla 2 se muestran los resultados para la concentración de $\mathrm{Hg}$ obtenida en las 5 muestras de lixiviados, así como los valores del coeficiente de partición $\left(K_{d}\right.$ y log $\left.K_{d}\right)$ y la fracción de metal disuelta y 
adsorbida. El coeficiente de partición para el mercurio en los lixiviados del relleno sanitario la Esmeralda estuvo entre 222.4 y 3460.2 L/kg, lo cual confirma la variabilidad en la composición de los mismos.

Es frecuente expresar el valor del logaritmo en base 10 del coeficiente de partición $\left(\log K_{d}\right)$, dado el amplio rango de valores que puede tomar $K_{d}$. Valores de $\log K_{d}$ menores a 2.0 se asocian con sustancias químicas completamente disueltas, en tanto que valores superiores a 5.0 indican que la especie se encuentra principalmente unida al material particulado (Thomann y Di Toro, 1983). El log $K_{d}$ para el mercurio en los lixiviados del relleno Sanitario La Esmeralda varió entre 2.35 y 3.54, el cual es un rango amplio que no permite generalizar sobre la afinidad del Hg hacia los sólidos suspendidos o hacia la fase acuosa. Los valores de log Kd para el $\mathrm{Hg}$ han sido determinados principalmente en arroyos, ríos y lagos, encontrándose valores de entre 2.2 - 5.8 (suelo/agua), 4.2 - 6.9 (materia suspendida/agua) y 3.8 - 6.0 (sedimento/agua) (Allison y Allison, 2005), pero para lixiviados de rellenos sanitarios no se dispone de información. Sin embargo, los valores de log Kd para el $\mathrm{Hg}$ en los lixiviados del relleno Sanitario La Esmeralda están en el rango reportado para este metal en otros sistemas. El lixiviado 5 presentó el menor valor del log
$K_{d}(2.35)$ y la mayor fracción disuelta de Hg (0.91), indicando que en esta muestra el Hg se encuentra principalmente disuelto. En contraste, el lixiviado 2 presentó el mayor valor de log Kd (3.54) y la menor fracción disuelta de $\mathrm{Hg}$ (0.18), sugiriendo que el $\mathrm{Hg}$ se encuentra esencialmente adsorbido en los sólidos. En los lixiviados 1 y 4 la concentración de SST es similar y la concentración de Hg total y disuelto difiere en un orden de magnitud de aprox. 9, sin embargo, el valor de $\log K_{d}$ y la fracción de metal disuelto y adsorbido son prácticamente iguales. El anterior resultado sugiere que existe un equilibrio de la cantidad disuelta y adsorbida del metal y la concentración de sólidos suspendidos (Thomann y Di Toro, 1983). Con relación al lixiviado 3 se observa que los sólidos suspendidos tienen una capacidad limitada de adsorción del mercurio, dado que esta muestra contenía la mayor concentración de metal, pero una baja cantidad de sólidos suspendidos.

La correlación entre la cantidad de sólidos suspendidos totales (log SST) presentes en el lixiviado y el $\log K_{d}$ (Figura 2), sugiere una interacción de las especies de mercurio con el material particulado, sin embargo, no es posible profundizar en aspecto, por cuanto no se determinó el tipo de especies de Hg presentes en el lixiviado ni las propiedades de los SST como carga y área superficial.

\begin{tabular}{|c|c|c|c|c|c|c|c|}
\hline Muestra & $\begin{array}{c}\text { Hg total } \\
(\mu \mathrm{g} / \mathrm{L})^{*}\end{array}$ & $\begin{array}{l}\text { Hg disuelto } \\
(\mu \mathrm{g} / \mathrm{L})^{*}\end{array}$ & $\begin{array}{c}\text { SST } \\
\text { (kg/L)* }\end{array}$ & $\mathrm{Kd}(\mathrm{L} / \mathrm{kg})$ & $\log K_{d}$ & $\mathbf{F}_{\text {dis }}$ & $\mathbf{F}_{\text {ads }}$ \\
\hline Lixiviado 1 & 102.20 & 23.30 & 0.001220 & 2775.6 & 3.44 & 0.23 & 0.77 \\
\hline Lixiviado 2 & 122.83 & 22.20 & 0.001310 & 3460.2 & 3.54 & 0.18 & 0.82 \\
\hline Lixiviado 3 & 1336.80 & 926.98 & 0.000640 & 690.8 & 2.84 & 0.69 & 0.31 \\
\hline Lixiviado 4 & 892.86 & 214.29 & 0.001230 & 2574.5 & 3.41 & 0.24 & 0.76 \\
\hline Lixiviado 5 & 71.40 & 65.01 & 0.000442 & 222.4 & 2.35 & 0.91 & 0.09 \\
\hline
\end{tabular}

Tabla 1. Concentración de $\mathrm{Hg}$ en los lixiviados y variables asociadas al coeficiente de partición. *Valor promedio de tres mediciones. 


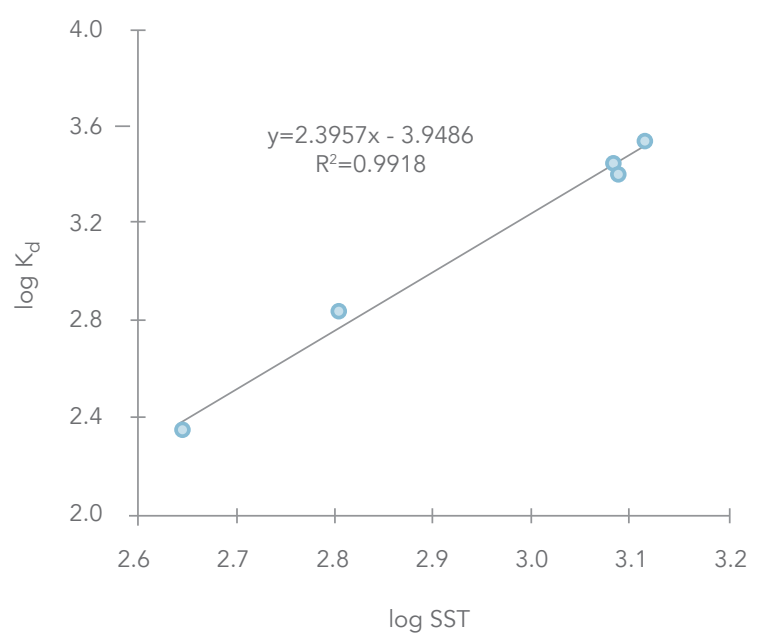

Figura 2. Variación del $\log K_{d}$ del mercurio con el contenido de sólidos suspendidos totales (log SST) presentes en los lixiviados del relleno sanitario La Esmeralda.

Para la muestra de lixiviado 4, la cual presentaba gran concentración sólidos suspendidos totales (1230 mg/L), así como una elevada concentración de $\mathrm{Hg}$ total y $\mathrm{Hg}$ disuelto (892.86 y $214.29 \mu \mathrm{g} / \mathrm{L}$ ), se realizó el análisis de variación del coeficiente de partición en función del pH de la muestra. En la Figura 3 se aprecia un incremento del $\log K_{d}$ con el aumento del $\mathrm{pH}$, el cual tiende a estabilizarse a $\mathrm{pH}$ mayor de 8.0. A pH bajos el mercurio se solubiliza y cuando el $\mathrm{pH}$ se incrementa el mercurio tiende a adsorberse en los sólidos suspendidos. Los anteriores resultados indican que la solubilidad y el equilibrio de adsorción - desorción de las especies de $\mathrm{Hg}$ dependen del $\mathrm{pH}$ del lixiviado. Para las otras muestras de lixiviado, el comportamiento de $\log K_{d}$ con el $\mathrm{pH}$ fue similar.

Teniendo en cuenta que la concentración total de mercurio presente en los lixiviados del relleno sanitario La Esmeralda se distribuye en las fases particulada y disuelta y que el coeficiente de partición varía con el pH de la muestra, un sistema de posttratamiento con materiales adsorbentes podría ser considerado para la remoción de este metal. La ventaja del proceso de adsorción es que se aprovecha una característica propia del metal de interés (afinidad por adsorberse en los sólidos), y es uno de los métodos más utilizados para el tratamiento de aguas residuales, dado su capacidad de retener contaminantes presentes en bajas concentraciones.

\section{CONCLUSIONES}

El agua residual doméstica a la que se adicionó una cantidad conocida de mercurio fue una matriz adecuada para la validación de la metodología de cuantificación de mercurio por espectroscopia de absorción atómica de vapor frío. El método mostró linealidad (coeficiente de correlación $=99.7 \%$ ), precisión (desviación estándar relativa $=4.49 \%$ ) y exactitud (error relativo promedio $=0.95 \%$ ) bajo las condiciones analíticas empleadas.

El coeficiente de partición de mercurio en los lixiviados del relleno sanitario La Esmeralda entre 222.4 y $3460.2 \mathrm{~L} / \mathrm{kg}$ se relaciona con la composición variable de los mismos, dado el amplio intervalo encontrado en la concentración de Hg total (71.4 - $1336.8 \mu \mathrm{g} / \mathrm{L}$ ) y sólidos suspendidos (442 - 1310

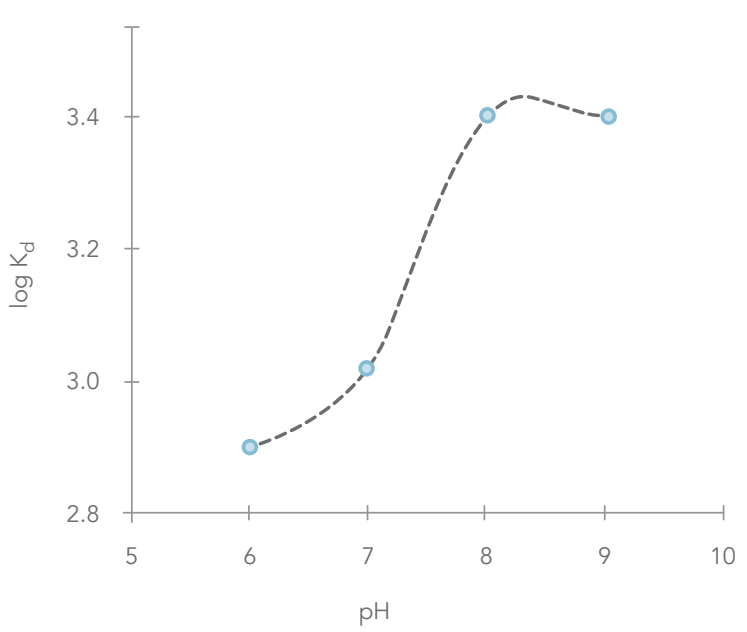

Figura 3. Variación de $\log \mathrm{K}_{\mathrm{d}}$ en función del pH para una muestra de lixiviado 4. 
$\mathrm{mg} / \mathrm{L})$. Valores de $\log \mathrm{K}_{\mathrm{d}}$ entre 2.35 y 2.84 se asocian a muestras de lixiviado en las cuales el $\mathrm{Hg}$ tiende a estar disuelto, en tanto que valores de log $\mathrm{K}_{d}$ entre 3.41 y 3.54 indican mayor afinidad del $\mathrm{Hg}$ a adsorberse sobre los sólidos suspendidos.

Para las muestras de lixiviado analizadas en este trabajo, el log $K_{d}$ varió linealmente con el log SST, resultado que sugiere interacción de las especies de $\mathrm{Hg}$ con el material particulado. Adicionalmente, cuando el pH del lixiviado disminuye a 6 , se modifica la distribución de las especies de mercurio en las dos fases, aumentando la concentración de mercurio disuelto ( $K_{d}$ disminuye). A pH superior a 8 , la relación de la concentración de $\mathrm{Hg}$ adsorbido y disuelto permanece constante ( $K_{d}$ no varía).

\section{AGRADECIMIENTOS}

Los autores agradecen a la Universidad Nacional de Colombia Sede Manizales (Proyecto 10658 - "Estudio de remoción de mercurio de aguas residuales provenientes de un relleno sanitario" - Convocatoria DIMA) por el apoyo financiero para el desarrollo del presente trabajo.

\section{REFERENCIAS}

1. Álvarez-Vazquez H., Jefferson B. y Judd S.J. 2004. Membrane bioreactors vs conventional biological treatment of landfill leachate: a brief review. Journal of Chemical Technology \& Biotechnology, 79: 1043-1049.

2. Allison J.D. y Allison T.L. 2005. Partition coefficients for metals in surface water, soil, and waste. EPA/600/R-05/074. Technical Report. U.S. Environmental Protection Agency. Washington D.C., 94 p.
3. ANLA. 2013. Metodología para la definición de la longitud de influencia de vertimientos sobre corrientes de agua superficial. Anexo 3. Autoridad Nacional de Licencias Ambientales. Subdirección de Instrumentos, Permisos y Trámites Ambientales. Bogotá. 25 p.

4. APHA, AWWA y WEF. 2005. Standard Methods for the Examination of Water and Wastewater. 21st edition, American Public Health Association, Washington D.C., 1200 p.

5. Calace N. 2001. Characteristics of different molecular weight fractions of organic matter in landfill leachate and their role in soil sorption of heavy metals. Environmental Pollution, 113: 331-339.

6. Claret F., Tournassat C., Crouzet C., Gaucher E.C., Schäfer T., Braibant G. y Guyonnet, D. 2011. Metal speciation in landfill leachates with a focus on the influence of organic matter. Waste Management, 31: 2036-2045.

7. da Cunha R.C., Patrício P.R., Vargas S.J.R., da Silva L.H.M. y da Silva M.C.H. 2016. Green recovery of mercury from domestic and industrial waste. Journal of Hazardous Materials, 304: 417-424.

8. Date Y., Aota A., Terakado S., Sasaki K., Matsumoto N., Watanabe Y., Matsue T. y Ohmura N. 2013. Trace-level mercury ion (Hg2+) analysis in aqueous sample based on solid-phase extraction followed by microfluidic immunoassay. Analytical Chemistry, 85: 434-440.

9. Ellison S.L. y Thompson M. 2008. Standard additions: myth and reality. Analyst, 133: 992-997.

10. Fan H.-J., Shu H.-Y., Yang H.-S. y Chen W.-C. 2006. Characteristics of landfill leachates in central Taiwan. Science of the Total Environment, 361: 25-37.

11. Fang, J.-J., Yang N., Cen, D.-Y., Shao, L.-M. y He, P.-J. 2012. Odor compounds from different sources of landfill: Characterization and source identification. Waste Management, 32: 1401-1410. 
12. Harris D.C. 2007. Cap. 5. Quality Assurance and Calibration Methods. p. 78-95. En: Quantitative Chemical Analysis. Seventh Edition. W.H. Freeman and Company, New York, 663 p.

13. Hennebert P., Avellan A., Yan J. y AguerreChariol O. 2013. Experimental evidence of colloids and nanoparticles presence from waste leachates. Waste Management, 33: 1870-1881.

14. Kjeldsen P., Barlaz M.A., Rooker A.P., Baun A., Ledin A. y Christensen T.H. 2002. Present and long - term composition of MSW landfill leachate: A review. Critical Reviews in Environmental Science \& Technology, 32: 297-336.

15. Leopold K., Foulkes M. y Worsfold P. 2010. Methods for the determination and speciation of mercury in natural waters - A review. Analytica Chimica Acta, 663: 127-138.

16. Londoño-Carvajal A., Marín-Arias J.E., González -Hoyos N.J. y Ocampo-López O.L. 2014. Estimación de produccipon de lixiviados en rellenos sanitarios. Universidad Nacional de Colombia Sede Manizales, Manizales, $192 \mathrm{p}$.

17. Ministerio de Ambiente y Desarrollo Sostenible. 2015. Decreto 1076 de 2015, por medio del cual se expide el Decreto Único Reglamentario del Sector Ambiente y Desarrollo Sostenible. Bogotá - República de Colombia, 654 p.

18. Morales C.J. 2007. Estudio para la remoción de metales pesados en los lixiviados de rellenos sanitarios. Trabajo de Grado, Especialización en Ingeniería Ambiental, Facultad de Ingeniería y Arquitectura. Universidad Nacional de Colombia, Manizales, $96 \mathrm{p}$.

19. Noguera K.M. y Olivero J.T. 2010. Los rellenos sanitarios en Latinoamérica: Caso colombiano. Revista de la Academia Colombiana de Ciencias Exactas, Físicas y Naturales, 34: 347-356.

20. Peng Y. 2013. Perspectives on technology for landfill leachate treatment. Arabian Journal of Chemistry. http://dx.doi.org/10.1016/j.arabjc.2013.09.031, In Press.
21. Poulain A.J., Garcia E., Amyot M., Campbell P.G.C. y Ariya P.A. 2007. Mercury distribution, partitioning and speciation in coastal vs. inland High Arctic snow. Geochimica et Cosmochimica Acta, 71: 3419-3431.

22. Raghab S.M., Abd El Meguid A.M. y Hegazi H.A. 2013. Treatment of leachate from municipal solid waste landfill. HBRC Journal, 9: 187-192.

23. Renoua S., Givaudan J.G., Poulain S., Dirassouyan F. y Moulin P. 2008. Landfill leachate treatment: Review and opportunity. Journal of Hazardous Materials, 150: 468-493.

24. Saldarriaga-Isaza A., Villegas-Palacio C. y Arango S. 2015. Phasing out mercury through collective action in artisanal gold mining: Evidence from a framed field experiment. Ecological Economics, 120: 406-415.

25. Silva T.F.C.V., Silva M.E.F., Cunha-Queda C.A., Fonseca A., Saraiva I., Boaventura R.A.R. y Vilar V.J.P. 2013. Sanitary landfill leachate treatment using combined solar photo-Fenton and biological oxidation processes at pre-industrial scale. Chemical Engineering Journal, 228: 850-866.

26. Thomann R.V. y Di Toro D.M. 1983. Physicochemical model of toxic substances in the Great Lakes. Journal of Great Lakes Research, 9: 474-496.

27. Žagar D., Petkovšek G., Rajar R., Sirnik N., Horvat M., Voudouri A., Kallos G. y Četina M. 2007. Modelling of mercury transport and transformations in the water compartment of the Mediterranean Sea. Marine Chemistry, 107: 64-88.

28. Žgajnar Gotvajn A., Zagorc-Končan J. y Cotman M. 2011. Fenton's oxidative treatment of municipal landfill leachate as an alternative to biological process. Desalination, 275 : 269-275. 